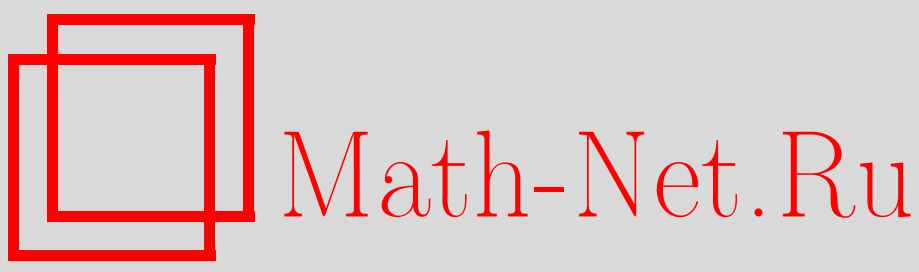

C. Е. Пастухова, Об усреднении одного вариационного неравенства для упругого тела с периодически расположенными трещинами, Матем. сб., 2000, том 191, номер 2, 149-164

DOI: https://doi.org/10.4213/sm456

Использование Общероссийского математического портала Math-Net.Ru подразумевает, что вы прочитали и согласны с пользовательским соглашением

http://www. mathnet.ru/rus/agreement

Параметры загрузки:

IP : 3.85 .73 .92

26 апреля 2023 г., 17:16:06 


\title{
С.Е. Пастухова \\ Об усреднении одного вариационного неравенства для упругого тела с периодически расположенными трещинами
}

\begin{abstract}
Изучена задача о малых деформациях упругого тела с периодически расположенньми трещинами, когда на краях трещин ставятся односторонние ограничения, которая эквивилентна вариационному неравенству. При условии, что $\varepsilon$ - линейный размер периода размещения трещин - стремится к нулю, доказана $L^{2}$-сходимость решений этой задачи к решению усредненной задачи, а ею является нелинейная краевая задача теории упругости в области без трещин.

Библиографйя: 13 названий.
\end{abstract}

\section{§1. Введение}

1. В $[1 ;$ гл. 6] была рассмотрена краевая задача для поля перемешений упругого тела, занимающего ограниченный трехмерный объем $\Omega$, с малыми периодически расположенными трещинами. На сторонах трещин накладывались односторонние ограничения: стороны трещин не перекрываются, на них отсутствует касательное трение, нормальное напряжение взаимно уравновешено на противоположных сторонах и оно ненулевое лиш в случае смыкания трещины. Известно, что такая краевая задача может быть поставлена в виде вариационного неравенства на некотором выпуклом замкнутом множестве функций (см. теорию вариационных неравенств в [2]-[4]). Если период распределения трешин, а также размеры их зависят от одного малого параметра $\varepsilon \rightarrow 0$, то, изучая поведение решения $u^{\varepsilon}$ этой краевой задачи (или вариационного неравенства) при $\varepsilon \rightarrow 0$, можно применить стандартный для теории усреднения метод асимптотических разложений (см. [1], а также похожие задачи в [5]-[7]). Формальное разложение для решения $u^{\varepsilon}$ выбирается в виде

$$
u^{\varepsilon}(x)=u^{0}(x)+\varepsilon u^{1}(x, y)+\varepsilon^{2} u^{2}(x, y)+\cdots, \quad y=\frac{x}{\varepsilon}, \quad x \in \Omega,
$$

где $u^{i}(x, y), i=1,2, \ldots$, как функции от $y=\left(y_{1}, y_{2}, y_{3}\right)$ являются 1-периодическими по всем переменным $y_{j}$. В [1] было показано, каким краевым задачам или вариационным неравенствам должны удовлетворять два первых члена этой асимптотики $u^{0}(x), u^{1}(x, y)$. Оказалось, как обычно бывает в теории усреднения, второй член асимптотики $u^{1}(x, y)$ определяет оператор краевой задачи для $u^{0}(x)$. Но в [1] остался открытым вопрос обоснования формальной асимптотики (1.1). Настоящая работа частично ликвидирует этот пробел, а именно, доказывается сходимость $u^{\varepsilon}(x)$ к $u^{0}(x)$ при $\varepsilon \rightarrow 0$ в $L^{2}$-норме. Таким образом, оказывается верной

$$
\text { (C) С.Е. Пастухова } 2000
$$


гипотеза, высказанная в [1]: упругое тело с периодически расположенньми трещинами, на сторонах которых действуют определенные односторонние ограничения, может рассматриваться как тело без трещин, для которого справедлив гиперупругий закон, связывающий тензоры напряжений и деформации.

2. Определим точно задачу о малых деформациях упругого тела с трещинами. Рассмотрим однородное упругое тело, занимающее ограниченную область $\Omega \in \mathbb{R}^{3}$ с гладкой (класса $C^{\infty}$ ) границей $\partial \Omega$. Его уравнение состояния имеет вид

$$
\sigma_{i j}(u)=a_{i j k h} e_{k h}(u)
$$

(здесь и далее по повторяющимся индексам подразумевается суммирование от 1 до 3$)$, где $u=\left(u_{1}, u_{2}, u_{3}\right)$ - поле перемещений, $e_{i j}(u), \sigma_{i j}(u)$ - тензоры деформации и напряжений соответственно, $e_{i j}(u)=\frac{1}{2}\left(\frac{\partial u_{i}}{\partial x_{j}}+\frac{\partial u_{j}}{\partial x_{i}}\right)$. Пусть коэффициенты тензора упругости $a=a_{i j k h}$ постоянны и обладают свойствами симметричности

$$
a_{i j k h}=a_{j i k h}=a_{i j h k}=a_{k h i j}
$$

и положительной определенности

$$
a_{i j k h} e_{i j} e_{k h} \geqslant \alpha e_{i j} e_{i j}
$$

для некоторой константы $\alpha>0$ и любого симметричного тензора $e=\left\{e_{i j}\right\}$, $e_{i j}=e_{j i}$.

Соотношение (1.2) можно обратить в следуюшем смысле: сушествует тензор $b=\left\{b_{i j k h}\right\}$ такой, что

$$
e_{i j}(u)=b_{i j k h} \sigma_{k h}(u)
$$

и для тензора $b$ вьполняются условия симметричности и положительной определенности, т.е.

$$
\begin{gathered}
b_{i j k h}=b_{j i k h}=b_{k h i j}=b_{i j h k} \\
b_{i j k h} \sigma_{i j} \sigma_{k h} \geqslant \alpha^{-1} \sigma_{i j} \sigma_{i j}
\end{gathered}
$$

для любого симметричного тензора $\sigma=\left\{\sigma_{i j}\right\}$.

Усложним ситуацию. В упругом теле появляется трещина или система трещин. Более точно это означает следуюшее. Пусть $F$ - замнутый кусок гладкой поверхности, $F \subset \Omega$. Он может быть несвязным, тогда $F$ - система нескольких или даже многих трещин. Основное требование: область $\Omega_{F}=\Omega \backslash F$ является связной, т.е. $F$ не разбивает объем $\Omega$ на несколько составляющих. Край поверхности $F$ состоит из конечного числа гладких кривых. Ясно, что область с трещиной $\Omega_{F}$ не имеет гладкой границы. В границу области $\Omega_{F}$ входят внешняя граница $\partial \Omega$, а также внутренняя граница, состоящая из двух экземпляров поверхности $F$, которые представляют две стороны трещины (или еше говорят: два берега трещины). Выберем на $F$ вектор единичной нормали $N=\left(N_{1}, N_{2}, N_{3}\right)$. Тогда ту сторону трещины, по отношению к которой вектор $N$ является внешней нормалью, будем обозначать через $F_{1}$, а другую сторону трещины - через $F_{2}$. Для функции $\varphi$, гладкой в $\Omega_{F}$, 
можно говорить о ее скачке на $F:[\varphi]=\left.\varphi\right|_{2}-\left.\varphi\right|_{1}$, где $\left.\varphi\right|_{i}=\left.\varphi\right|_{F_{i}}, i=1,2$. Для негладкой функции $\varphi \in H^{1}\left(\Omega_{F}\right)$ стандартным образом можно ввести два следа ее $\left.\varphi\right|_{i}=\left.\varphi\right|_{F_{i}}, i=1,2$, через которые так же, как для гладкой функции, определяется скачок $[\varphi]$ на поверхности $F$.

Пусть $n=\left(n_{1}, n_{2}, n_{3}\right)$ - вектор внешней единичной нормали к границе $\partial \Omega_{F}$. Тогда на границе $\partial \Omega_{F}$ действует напряжение, вектор которого $\sigma_{n}=\sigma n$ имеет компоненты $\sigma_{i j} n_{j}, i=1,2,3$, и раскладывается на взаимно ортогональные составляющие

$$
\sigma_{n}=\sigma_{n n} n+\sigma_{\tau},
$$

где $\sigma_{\tau}-$ касательная к границе составляющая.

Далее будем иметь в виду, что в силу введенных обозначений для двух сторон трещины $\left.n\right|_{F_{1}}=N,\left.n\right|_{F_{2}}=-N$.

Пусть выполнены следующие предположения. Две стороны трещины могут быть разомкнуты, но не могут перекрываться. В рассматриваемой математической модели разомкнутость трещины в какой-то точке $F$ означает, что на сторонах $F_{1}, F_{2}$ в этой точке нормальные перемещения (проекции перемещения на нормаль $N$ ) не совпадают, т.е. претерпевают скачок, неотрицательность которого интерпретируется как условие неперекрываемости сторон трещины. Аналогично считается, что трещина в некоторой точке смыкается, если нормальные перемещения в этой точке на сторонах $F_{1}, F_{2}$ совпадают. Далее, предположим также, что напряжения на сторонах трещины не содержат касательных компонент и взаимно уравновешиваются. Если трещина разомкнута в некоторой точке, то напряжения на обеих сторонах в этой точке вовсе отсутствуют. В точках смыкания трешины происходит сжатие тела. Предположим также, что тело закреплено на внешней границе $\partial \Omega$ и на него воздействуют внешние силы, вектор объемной плотности которых есть $f=\left(f_{1}, f_{2}, f_{3}\right)$.

В этих предположениях на упругое тело, занимающее объем $\Omega_{F}$, его поле перемещений $u=\left(u_{1}, u_{2}, u_{3}\right)$ удовлетворяет следуюшей краевой задаче в $\Omega_{F}$ с частично односторонними условиями на границе $\partial \Omega_{F}$ :

$$
\begin{gathered}
\operatorname{div} \sigma(u)+f=0 \text { в } \Omega_{F}, \\
u=0 \text { на } \partial \Omega, \\
{[u \cdot N] \geqslant 0 \text { на } F,} \\
\left.\sigma_{n}(u)\right|_{1}=\left.\sigma_{N N}\right|_{1} N,\left.\quad \sigma_{n}(u)\right|_{2}=-\left.\sigma_{N N}\right|_{1} N,\left.\quad \sigma_{N N}\right|_{1} \leqslant 0, \\
\left.\sigma_{N N}\right|_{1}[u \cdot N]=0 \text { на } F .
\end{gathered}
$$

Здесь и далее для векторов $u, v \in \mathbb{R}^{3}$ через $u \cdot v$ обозначаем их скалярное произведение, а также для тензора $\sigma=\left\{\sigma_{i j}\right\}$ считаем, что $\operatorname{div} \sigma=\frac{\partial}{\partial x_{j}} \sigma_{i j}$.

Как в [1; гл. 6], для обобщенной (вариационной) постановки задачи (1.5)-(1.9) введем в стандартном трехмерном соболевском пространстве $\left(H^{1}\left(\Omega_{F}\right)\right)^{3}$ : подпространство $V_{F}$ как замыкание множества функций из $\left(C_{\partial \Omega}^{\infty}\left(\Omega_{F}\right)\right)^{3}$, равных нулю в некоторой окрестности границы $\partial \Omega$, по норме $\left(H^{1}(\Omega)\right)^{3}$; множество функций $K_{F}=\left\{u \in V_{F}:[u \cdot N] \geqslant 0\right.$ п.в. на $\left.F\right\} ;$ билинейную форму $a(u, v)=\int_{\Omega} \sigma(u) e(v) d x$; 
линейную форму $L(u)=\int_{\Omega} u \cdot f d x$. Здесь и далее для тензоров $\sigma(u), e(v)$ используем обозначение $\sigma(u) e(v)=\sigma_{i j}(u) e_{i j}(v)$.

Известно (см. [1]), что $V_{F}$, снабженное обычной нормой из $\left(H^{1}(\Omega)\right)^{3}$, является гильбертовым пространством, а $K_{F}$ - замкнутым выпуклым конусом из $V_{F}$.

Тогда вариационная постановка задачи (1.5)-(1.9) такова: найти функиию $u \in K_{F}$, удовлетворяющую для любой $v \in K_{F}$ неравенству

$$
a(u, v-u) \geqslant L(v-u) .
$$

В $[1 ;$ гл. $6, \S 5]$ показана эквивалентность краевой задачи (1.5)-(1.9) и вариационного неравенства (1.10), если $f$ и и достаточно гладкие. Поэтому далее под обобшенньм решением краевой задачи (1.5)-(1.9) понимаем функцию $u \in K_{F}-$ решение вариационного неравенства (1.10) с заданной функцией $f \in\left(H^{-1}\left(\Omega_{F}\right)\right)^{3}$.

В области $\Omega_{F}$ выполняется неравенство Корна, с помощью которого в силу (1.3) выводится коэрцитивность билинейной формы $a(u, v)$ на пространстве $V_{F}$. И значит (см. [1; гл. 6, теорема 5.1]), существует и притом единственное решение вариационного неравенства (1.10).

3. Рассмотрим последовательность зависящих от малого параметра $\varepsilon(0<\varepsilon<1)$ задач типа задачи (1.10), но сперва определим необходимые множества. Пусть $Y=(0,1)^{3}$ - единичный куб в $\mathbb{R}^{3}$. В $Y$ есть гладкая “трещина" $F$ в смысле п. 2 , не пересекающая границу куба $Y$. Пусть $Y_{F}=Y \backslash F$, а $F_{0}$ есть неограниченное множество с 1-периодической структурой такое, что $F_{0} \cap Y=F$.

Пусть $F_{\varepsilon 0}=\varepsilon F_{0}=\left\{x \in \mathbb{R}^{3}: \varepsilon^{-1} x \in F_{0}\right\}$. Если $T_{\varepsilon}=\left\{z \in \mathbb{Z}^{3}: \varepsilon(\bar{Q}+z) \in \Omega\right\}$, где $Q=(0,1)^{3}$ - единичньй куб в $\mathbb{R}^{3}$, то множество $F_{\varepsilon}=\bigcup_{z \in T_{\varepsilon}} \varepsilon(F+z)$ есть некоторая часть $F_{\varepsilon 0}$, попавшая в область $\Omega$, притом не выходящая на границу $\partial \Omega$. Множество $F_{\varepsilon}$ будем рассматривать как систему периодически (с периодом $\varepsilon Y$ ) расположенных в $\Omega$ трещин. Пусть $\Omega_{\varepsilon}=\Omega \backslash F_{\varepsilon}$. Тогда граница области $\Omega_{\varepsilon}$ состоит из внешней гранищы $\partial \Omega$ и двух экземпляров $F_{\varepsilon, 1}, F_{\varepsilon, 2}$ множества $F_{\varepsilon}$, представляющих две стороны (два берега) системы трещин $F_{\varepsilon}$ в $\Omega$. Пусть обозначение двух сторон этой системы трещин через $F_{\varepsilon, 1}, F_{\varepsilon, 2}$ соответствует обозначению $F_{1}, F_{2}$ двух сторон трещины $F$ в $Y$, порождающей систему трещин $F_{\varepsilon}$ описанным выше способом. Единичную нормаль к $F$, которая является внешней на стороне $F_{1}$, обозначаем через $N$, так же будем обозначать ту единичную нормаль к $F_{\varepsilon}$, которая является внешней на $F_{\varepsilon, 1}$.

Пусть для функции $\varphi$, определенной на $\Omega_{\varepsilon}$ (или на $\left.Y_{F}\right),[\varphi]=\left.\varphi\right|_{2}-\left.\varphi\right|_{1}$, где $\left.\varphi\right|_{i}=\left.\varphi\right|_{F_{\varepsilon, i}}\left(\right.$ или $\left.\left.\varphi\right|_{i}=\left.\varphi\right|_{F_{i}}\right), i=1,2$.

Определим пространство $V_{\varepsilon}=V_{F_{\varepsilon}}$ и выпуклое множество $K_{\varepsilon}=K_{F_{\varepsilon}}$ аналогично тому, как в п. 2 были определены $V_{F}$ и $K_{F}$, взяв вместо области $\Omega_{F}$ область $\Omega_{\varepsilon}$. Пусть теперь $a(u, v)=\int_{\Omega_{\varepsilon}} \sigma(u) e(v) d x, L(u)=\int_{\Omega_{\varepsilon}} u \cdot f d x$ для любых функций $u, v \in V_{\varepsilon}$ и фиксированной $f \in\left(L^{2}(\Omega)\right)^{3}$.

Под решением статической задачи для малых деформаций упругого тела с трещинами, занимаюшего объем $\Omega_{\varepsilon}$, понимаем вектор-функцию $u^{\varepsilon} \in K_{\varepsilon}$, для которой неравенство

$$
a\left(u^{\varepsilon}, v-u^{\varepsilon}\right) \geqslant L\left(v-u^{\varepsilon}\right)
$$

справедливо при любой функции $v \in K_{\varepsilon}$. 
В пространстве $V_{\varepsilon}$ выполнено равномерное неравенство Корна

$$
\int_{\Omega_{\varepsilon}}\left(|\nabla u|^{2}+|u|^{2}\right) d x \leqslant C \int_{\Omega_{\varepsilon}}|e(u)|^{2} d x
$$

где константа $C$ одна и та же для всех $u \in V_{\varepsilon}$ и всех $\varepsilon$, достаточно малых $\left(\varepsilon<\varepsilon_{0}\right)$. Неравенство (1.12) можно вывести из соответствуюшего равномерного неравенства Корна для периодически перфорированных областей (см., например, [6]).

Используя (1.12), стандартно можно доказать существование решения вариационного неравенства (1.11), которое является единственным и удовлетворяет оценке

$$
\left|u^{\varepsilon}\right|_{H^{1}(\Omega)^{3}} \leqslant C_{1} \quad\left(\varepsilon<\varepsilon_{0}\right)
$$

где константа $C_{1}$ не зависит от $\varepsilon$.

Если подвектором $n=\left(n_{1}, n_{2}, n_{3}\right)$ понимать единичную внешнюю нормаль к $F_{\varepsilon}$, то так же, как в п. 2, определим для заданного поля перемещений $u=\left(u_{1}, u_{2}, u_{3}\right)$ на $F_{\varepsilon}$ вектор напряжений $\sigma_{n}=\sigma(u) n$, при этом $\sigma_{n}$ раскладьвается в сумму взаимно ортогональных компонент типа суммы (1.4).

Вариационное неравенство (1.11) является обобщенной постановкой краевой задачи

$$
\begin{gathered}
\operatorname{div} \sigma\left(u^{\varepsilon}\right)+f=0 \text { в } \Omega_{\varepsilon} \\
u^{\varepsilon}=0 \text { на } \partial \Omega, \quad\left[u^{\varepsilon} \cdot N\right] \geqslant 0 \text { на } F_{\varepsilon}, \\
\left.\sigma_{n}\left(u^{\varepsilon}\right)\right|_{1}=\left.\sigma_{N N}\left(u^{\varepsilon}\right)\right|_{1} N,\left.\quad \sigma_{n}\left(u^{\varepsilon}\right)\right|_{2}=-\left.\sigma_{N N}\left(u^{\varepsilon}\right)\right|_{1} N,\left.\quad \sigma_{N N}\left(u^{\varepsilon}\right)\right|_{1} \leqslant 0 \\
\left.\sigma_{N N}\left(u^{\varepsilon}\right)\right|_{1}\left[u^{\varepsilon} \cdot N\right]=0 \text { на } F_{\varepsilon} .
\end{gathered}
$$

4. Изучим поведение $u_{\varepsilon}$ при $\varepsilon \rightarrow 0$, где $u^{\varepsilon}$ - решение неравенства (1.11). Для этого будем искать решение $u^{\varepsilon}$ в виде разложения (1.1) и выбирать пробные функции $v^{\varepsilon}$ в неравенстве (1.11) в виде аналогичного разложения:

$$
v^{\varepsilon}=v^{0}(x)+\varepsilon v^{1}(x, y)+\varepsilon^{2} v^{2}(x, y)+\cdots
$$

В (1.1) и (1.14) $u^{i}(x, y), v^{i}(x, y), i=1,2 \ldots,-$ вектор-функции, определенные на $\Omega \times Y_{F}, 1$-периодические по $y$. Так как $u^{0}(x), v^{0}(x)$ не зависят от $y$, то условие $u^{\varepsilon} \in K_{\varepsilon}$ означает, что

$$
\left[u^{1} \cdot N\right] \geqslant 0 \text { п.в. на } F \text {. }
$$

Введем теперь необходимые пространства и множества функций на ячейке $Y_{F}$, используя обозначения из [1]: $\quad V_{Y F}=\left\{v=\left(v_{1}, v_{2}, v_{3}\right): v_{i} \in H^{1}\left(Y_{F}\right), v_{i} 1\right.$-периодические по $y, i=1,2,3\}, \widetilde{V}_{Y F}=\left\{v \in V_{Y F}: \bar{v}=0\right\}$, где $\bar{v}=\int_{Y} v d y$, $K_{Y F}=\left\{v \in V_{Y F}:[v \cdot N] \geqslant 0\right.$ п.в. на $\left.F\right\}, \widetilde{K}_{Y F}=\left\{v \in K_{Y F}: \bar{v}=0\right\}$.

Используя эти обозначения, а также соображение (1.15), можем считать, что в разложениях $(1.1),(1.14) u^{0}, v^{0} \in\left(H_{0}^{1}(\Omega)\right)^{3}$, а $u^{1}, v^{1}$ - функции по $x$ со значениями в $K_{Y F}$. 
В [1; гл. 6, предложение 6.1] показано, что, рассматривая неравенство (1.11) для функций $u^{\varepsilon}$ и $v=v^{\varepsilon}$ вида (1.1), (1.14), можно вьвести следующие свойства функций $u^{0}, u^{1}$ из разложения (1.1). Функция $u^{0}$ принадлежит $\left(H_{0}^{1}(\Omega)\right)^{3}$ и удовлетворяет вариационному равенству

$$
\int_{\Omega} \int_{Y} a_{i j k h}\left(\frac{\partial u_{k}^{0}}{\partial x_{h}}+\frac{\partial u_{k}^{1}}{\partial y_{h}}\right) \frac{\partial}{\partial x_{j}}\left(v_{i}-u_{i}^{0}\right) d x d y-\int_{\Omega} f_{i}\left(v_{i}-u_{i}^{0}\right) d x=0
$$

для любой функции $v \in\left(H_{0}^{1}(\Omega)\right)^{3}$. А это эквивалентно тому, что $u^{0}$ - решение следуюшей краевой задачи в области $\Omega$ :

$$
\operatorname{div} \bar{\sigma}^{0}\left(u^{0}\right)+f=0 \quad \text { в } \Omega, \quad u^{0} \in\left(H_{0}^{1}(\Omega)\right)^{3},
$$

где

$$
\begin{aligned}
\bar{\sigma}^{0} & =\int_{Y} \sigma^{0} d y, & \sigma^{0} & =\left\{\sigma_{i j}^{0}\right\} \\
\sigma_{i j}^{0} & =\sigma_{i j}^{0}\left(u^{0}\right)=a_{i j k h}\left(\frac{\partial u_{k}^{0}}{\partial x_{h}}+\frac{\partial u_{k}^{1}}{\partial y_{h}}\right), & i, j & =1,2,3,
\end{aligned}
$$

что можно переписать по-иному ввиду симметричности тензоров $a, e(u)$ :

$$
\sigma_{i j}^{0}(E)=a_{i j k h}\left(E_{k h}+e_{k h, y}\left(u^{1}\right)\right), \quad i, j=1,2,3,
$$

где $E_{k h}=e_{k h}\left(u^{0}\right)$, тензор деформации $e_{i j, y}(v)$ для заданного перемешения $v$ определяется через $\operatorname{grad}_{y} v(y)$ стандартньм образом. При этом функция $u^{1}(x, y)$ принадлежит $K_{Y F}$ и при фиксированных $x, u^{0}(x)$ удовлетворяет вариационному неравенству для любой функции $w \in K_{Y F}$ :

$$
\int_{Y} a_{i j k h}\left(\frac{\partial u_{k}^{0}}{\partial x_{h}}+\frac{\partial u_{k}^{1}}{\partial y_{h}}\right) \frac{\partial}{\partial y_{j}}\left(w_{i}-u_{i}^{1}\right) d y \geqslant 0 .
$$

Заметим, что вектор-функции $u^{1}, w$ входят в неравенство (1.19) через свой градиент по переменной $y$, поэтому каждую из них можно заменить на постоянньй вектор, отчего левая часть неравенства (1.19) не изменится, но измененные функции окажутся из конуса $\widetilde{K}_{Y F}$.

Введем на пространстве $V_{Y F}$ билинейную форму

$$
a_{Y}(v, w)=\int_{Y} a_{i j k h} e_{i j, y}(v) e_{k h, y}(w) d y,
$$

а также линейную форму, зависяшую от тензорного параметра $E=\left\{E_{i j}\right\}$ :

$$
L_{E}(v)=\int_{Y} a_{i j k h} E_{i j} e_{k h, y}(v) d y .
$$

Учтем, что ввиду симметричности тензоров $a, e(v)$ есть другое представление формы (1.20), а именно

$$
a_{Y}(v, w)=\int_{Y} a_{i j k h} \frac{\partial v_{k}}{\partial y_{h}} \frac{\partial w_{i}}{\partial y_{j}} d y .
$$


Из неравенства Корна на ячейке $Y_{F}$ можно вывести коэрцитивность формы $a_{Y}(v, w)$ на $\widetilde{V}_{Y F}$, и это свойство обеспечит разрешимость, и притом однозначную, локальной задачи на ячейке $Y_{F}$ для отыскания функции $u^{1}(x, y)$, которую с учетом (1.19)-(1.22) можно сформулировать в следующем виде.

Пусть $u^{0}(x)$ - заданная функция. Сопоставим ей тензор $E=\left\{E_{i j}\right\}, E_{i j}=$ $e_{i j}\left(u^{0}\right)$. Тогда $u^{1}(x, y)$ - решение следуюшей задачи: найти функиию $u \in \widetilde{K}_{Y F}$, для которой неравенство

$$
a_{Y}(u, w-u)+L_{E}(w-u) \geqslant 0
$$

выполняется при любой функиии $w \in \widetilde{K}_{Y F}$.

Теорема 1 (см. [1; гл. 6, теорема 6.1]). Задача (1.23) имеет единственное решение в $\widetilde{K}_{Y F}$ для заданного тензора $E=\left\{E_{i j}\right\}$.

В силу теоремы 1 тензор $\bar{\sigma}^{0}=\left\{\bar{\sigma}_{i j}^{0}\right\}$, определенный формулами (1.17), (1.18), является нелинейной функцией от аргумента $E=\left\{E_{k h}\right\}, E_{k h}=e_{k h}\left(u^{0}\right)$ (который можно рассматривать как элемент из $\mathbb{R}^{6}$ в силу его симметричности), при этом отображение $E \rightarrow \bar{\sigma}^{0}$ задается формулами (см. [1; гл. 6, теорема 7.1]):

$$
\begin{gathered}
\bar{\sigma}_{i j}^{0}=\frac{\partial W}{\partial E_{i j}} \\
W(E)=\frac{1}{2} \int_{Y} a_{i j k h}\left(e_{k h, y}(u)+E_{k h}\right)\left(e_{i j, y}(u)+E_{i j}\right) d y
\end{gathered}
$$

где $u$ - решение неравенства (1.23) при заданном тензоре $E=\left\{E_{i j}\right\}$.

В теоремах 7.1, $7.2\left[1 ;\right.$ гл. 6] приведены свойства функций $\bar{\sigma}^{0}(E), W(E)$, зависящих от тензорного аргумента $E$. Из этих свойств на основании результатов [8] вьводится обобщенная разрешимость, притом однозначная, нелинейной краевой задачи (1.16).

Под обобшенным решением задачи (1.16) понимаем функцию $u^{0} \in\left(H_{0}^{1}(\Omega)\right)^{3}$ такую, что для любой функции $w \in\left(H_{0}^{1}(\Omega)\right)^{3}$ выполнено равенство

$$
\int_{\Omega} \bar{\sigma}_{i j}^{0}(E) e_{i j}(w) d x=\int_{\Omega} f_{i} w_{i} d x, \quad \text { где } E=\left\{E_{k h}\right\}, \quad E_{k h}=e_{k h}\left(u^{0}\right) .
$$

Tеорема 2 (см. [1; гл. 6, теорема 7.3]). Для любой заданной функции $f \in$ $\left(H^{-1}(\Omega)\right)^{3}$ существует и притом единственное обобщенное решение задачи (1.16).

\section{§ 2. Обоснование процедуры усреднения задачи (1.11)}

Основной результат работы дан в теореме 4, которая является следствием теоремњ 3. Но прежде чем перейти к этим теоремам, дадим в п. 1 ряд вспомогательных утверждений.

1. В дальнейшем будем использовать некоторые приемы из работ [9]-[12], [7; гл. 16], которые успешно применялись ранее при усреднении различных линейных 
и нелинейных задач в перфорированных областях. Среди них особо отметим: модифицированную лемму о компенсированной компактности (обычную лемму о компенсированной компактности см., например, в [7; гл. $1, \S 1])$, обращение к двойственным функционалам, кусочно аффинное приближение предельной функции.

Введем необходимые пространства для вспомогательных лемм. Пусть $C_{\text {per }}^{\infty}\left(Y_{F}\right)$ является множеством гладких 1-периодических функций, определенных на $\mathbb{R}^{3} \backslash F_{0}$, тогда $L_{\mathrm{per}}^{2}\left(Y_{F}\right)$ есть замыкание $C_{\mathrm{per}}^{\infty}\left(Y_{F}\right)$ по норме $L^{2}(Y)$. Определим $H_{\mathrm{per}}^{1}(Y, F)$ как замыкание подмножества функций из $C_{\text {per }}^{\infty}\left(Y_{F}\right)$, равных 0 в окрестности $F_{0}$, по норме $H^{1}(Y)$.

Лемма 1. Пусть $a(y) \in L_{\mathrm{per}}^{2}\left(Y_{F}\right), \bar{a}=\int_{Y} a d y=0$. Тогда существует $w \in H_{\mathrm{per}}^{1}(Y, F)^{3}$ такая, что $\operatorname{div} w(y)=a(y)$, при этом

$$
|w|_{H^{1}(Y)^{3}} \leqslant C_{0}|a|_{L^{2}(Y)}, \quad \text { əde } \quad C_{0}=\operatorname{const}(F) .
$$

Доказательство для случая, когда $F$ является полостью в $Y$ с $C^{2}$-границей, дано в $[5 ;$ гл. $4, \S 7]$. С учетом результатов работы $[13]$ нужный нам случай рассматривается аналогично.

ЛЕмма 2. Пусть $u^{(i)}$ - решение вариационного неравенства (1.23) для заданного тензора $E^{(i)}=\left\{E_{k h}^{(i)}\right\}, i=1,2$. Тогда

$$
\int_{Y}\left|e\left(u^{(1)}-u^{(2)}\right)\right|^{2} d y \leqslant C_{1} \int_{Y}\left|E^{(1)}-E^{(2)}\right|^{2} d y, \quad \text { əde } \quad \mathrm{C}_{1}=\operatorname{const}(F) .
$$

Доказательство последней оценки можно провести следующим образом: записать неравенства, которым функции $u^{(1)}, u^{(2)}$ как решения соответствуюших вариационных задач удовлетворяют с пробньми функциями $v=u^{(2)}, v=u^{(1)}$ соответственно, далее сложить эти неравенства, и стандартные преобразования приведут к нужной оценке.

Далее для функции $v(y) \in V_{Y F}$ под $e_{k h}(v)$ подразумеваем $e_{k h, y}(v)$.

Лемма 3. Если $u_{E}(y)$ - решение вариационного неравенства (1.23) с заданным тензором $E=\left\{E_{i j}\right\}$, компоненты которого постоянны, то тензорная функиия $z=\left\{z_{i j}\right\}$ такая, что $z_{i j}=a_{i j k h}\left(E_{k h}+e_{k h}\left(u_{E}\right)\right)$, имеет следующие свойства:

$$
\operatorname{div} z=0 \quad \text { в } \quad Y_{F}
$$

на границе $F$ взаимно ортогональное разложение вектора $z_{n}=z n=z_{n n} n+z_{\tau}$ удовлетворяет условиям:

$$
\begin{gathered}
\left.z_{n}\right|_{1}=\left.z_{n n}\right|_{1} N,\left.\quad z_{n}\right|_{2}=-\left.z_{n n}\right|_{1} N,\left.\quad z_{n n}\right|_{1} \leqslant 0 \\
\int_{Y_{F}} a_{i j k h}\left(E_{i j}+e_{i j}\left(u_{E}\right)\right)\left(E_{k h}+e_{k h}\left(u_{E}\right)\right) d y=\bar{\sigma}^{0}(E) E=\bar{z} E
\end{gathered}
$$

əде $\bar{\sigma}^{0}(E)=\bar{z}=\int_{Y} z d y$. 
Доказательство свойств (2.1), (2.2) проводится так же, как в [1; гл. 6] доказывались свойства (1.5)-(1.9) для решения вариационного неравенства (1.10). Свойство (2.3) вытекает из неравенств, которым удовлетворяет $u_{E}$ как решение соответствующей вариационной задачи с пробньми функциями $v=0, v=2 u_{E}$.

Пусть дана последовательность $u^{\varepsilon} \in V_{\varepsilon}$ такая, что

$$
\varlimsup_{\varepsilon \rightarrow 0} \int_{\Omega}\left|u^{\varepsilon}\right|^{2} d x \leqslant+\infty, \quad \varlimsup_{\varepsilon \rightarrow 0} \int_{\Omega}\left|\nabla u^{\varepsilon}\right|^{2} d x \leqslant+\infty .
$$

Тогда без потери общности можно считать, что

$$
u^{\varepsilon} \rightarrow u^{0} \text { слабо в } L^{2}(\Omega) .
$$

ЛЕмма 4. Пусть последовательность $u^{\varepsilon} \in V_{\varepsilon}$ удовлетворяет условия.м (2.4), (2.5), тогда $u^{0} \in\left(H_{0}^{1}(\Omega)\right)^{3} u$

$$
\int_{\Omega}\left|\nabla u^{0}\right|^{2} d x \leqslant c_{0} \varlimsup_{\varepsilon \rightarrow 0} \int_{\Omega}\left|\nabla u^{\varepsilon}\right|^{2} d x
$$

Доказательство этой леммы дано в [12; с. 398], идеи его можно найти также в $[10]$.

Лемма 5. Пусть $u^{\varepsilon} \in V_{\varepsilon}$ есть решение задачи (1.11), и пусть $z^{\varepsilon}(x)=$ $z(x / \varepsilon)$, где $z(y)=\left\{z_{i j}(y)\right\}-$ тензор, определенный в лемме 3 . Тогда последовательность и у $^{\varepsilon}$ довлетворяет условиям (2.4), (2.5) и имеет место оценка

$$
\varliminf_{\varepsilon \rightarrow 0} \int_{A} \varphi \frac{\partial u_{i}^{\varepsilon}}{\partial x_{j}} z_{i j}^{\varepsilon}(x) d x \geqslant \bar{z}_{i j} \int_{A} \varphi \frac{\partial u_{i}^{0}}{\partial x_{j}} d x
$$

для любой подобласти $A \Subset \Omega$ и юбой неотрицательной функиии $\varphi \in C_{0}^{\infty}(A)$.

ДокАЗАТЕЛЬСТво. Поскольку для решения $u^{\varepsilon}$ задачи $(1.11)$ при различных $\varepsilon$ имеется равномерная оценка (1.13), то последовательность $u^{\varepsilon}$ действительно удовлетворяет условиям (2.4), (2.5). Заметим также, что $u^{\varepsilon} \in K_{\varepsilon}$, т.е. $u^{\varepsilon} \geqslant 0$ п.в. на $F_{\varepsilon}$.

Используя свойства (2.1), (2.2) тензора $z(y)$, приведенные в лемме 3 , имеем для интересующего нас интеграла, взятого по произвольной подобласти $A \Subset \Omega$, следующее представление:

$$
\begin{aligned}
I(\varepsilon)= & \int_{A} \varphi\left(\nabla u^{\varepsilon} \cdot z^{\varepsilon}\right) d x=-\int_{A \backslash F_{\varepsilon}}(\nabla \varphi) z^{\varepsilon} u^{\varepsilon} d x \\
& -\left.\left.\int_{A \cap F_{\varepsilon, 1}} \varphi z_{n n}\right|_{1}\left(u^{\varepsilon} \cdot N\right)\right|_{1} d \sigma-\left.\left.\int_{A \cap F_{\varepsilon, 2}} \varphi z_{n n}\right|_{1}\left(u^{\varepsilon} \cdot N\right)\right|_{2} d \sigma \\
= & -\int_{A \backslash F_{\varepsilon}}(\nabla \varphi) z^{\varepsilon} u^{\varepsilon} d x+\int_{A \cap F_{\varepsilon}} \varphi\left(-\left.z_{n n}\right|_{1}\right)\left[u^{\varepsilon} \cdot N\right] d \sigma .
\end{aligned}
$$


Ввиду неотрицательности всех трех сомножителей, стоящих в последнем интеграле из суммы (2.7), выводим неравенство

$$
\begin{aligned}
I(\varepsilon) & \geqslant-\int_{A \backslash F_{\varepsilon}}(\nabla \varphi) z^{\varepsilon} u^{\varepsilon} d x \\
& =\int_{A \backslash F_{\varepsilon}}(\nabla \varphi)\left(z^{\varepsilon}-\bar{z}\right) u^{\varepsilon} d x-\bar{z} \int_{A}(\nabla \varphi) u^{\varepsilon} d x=I_{1}(\varepsilon)+I_{2}(\varepsilon) .
\end{aligned}
$$

Найдем $\lim _{\varepsilon \rightarrow 0} I_{i}(\varepsilon), i=1,2$.

Так как каждая компонента функции $z(y)-\bar{z}$ удовлетворяет условиям леммы 1 , то найдется функция $w(y) \in H_{\mathrm{per}}^{1}(Y, F)^{3 \times 3 \times 3}$ такая, что $\operatorname{div}_{y} w(y)=z(y)-\bar{z}$ и ее $H^{1}$-норма ограничена величиной $C|z|_{L^{2}(Y)^{3 \times 3}}, C=\operatorname{const}(F)$. Отсюда получаем представление

$$
z^{\varepsilon}(x)-\bar{z}=\left.\left(\operatorname{div}_{y} w(y)\right)\right|_{y=x / \varepsilon}=\varepsilon \operatorname{div}_{x} w\left(\frac{x}{\varepsilon}\right) .
$$

Поэтому

$$
\begin{aligned}
I_{1}(\varepsilon) & =-\varepsilon \int_{A \backslash F_{\varepsilon}}(\nabla \varphi)\left(\operatorname{div}_{x} w\left(\frac{x}{\varepsilon}\right)\right) u^{\varepsilon} d x \\
& =\varepsilon \int_{A \backslash F_{\varepsilon}}(\nabla \nabla \varphi) w\left(\frac{x}{\varepsilon}\right) u^{\varepsilon} d x+\varepsilon \int_{A \backslash F_{\varepsilon}}(\nabla \varphi) w\left(\frac{x}{\varepsilon}\right) \nabla u^{\varepsilon} d x
\end{aligned}
$$

и, значит,

$$
I_{1}(\varepsilon) \rightarrow 0 \text { при } \varepsilon \rightarrow 0
$$

в силу условия (2.4) для функции $u^{\varepsilon}$ и оценки

$$
\int_{A \backslash F_{\varepsilon}}\left|w\left(\frac{x}{\varepsilon}\right)\right|^{2} d x \leqslant c_{1} \int_{Y_{F}}|w(y)|^{2} d y
$$

где константа $c_{1}$ не зависит от $\varepsilon\left(\varepsilon<\varepsilon^{0}\right)$.

Далее, используя условие (2.5) для последовательности $u^{\varepsilon}$, получаем

$$
I_{2}(\varepsilon)=-\bar{z} \int_{A}(\nabla \varphi) u^{\varepsilon} d x \rightarrow-\bar{z} \int_{A}(\nabla \varphi) u^{0} d x=\bar{z} \int_{A} \varphi \nabla u^{0} d x .
$$

Из (2.7)-(2.10) вытекает (2.6).

2. Введем следующие функционалы:

$$
\begin{aligned}
& J_{\varepsilon}(v)=\frac{1}{2} a(v, v)-L(v) \quad \text { для } v \in V_{\varepsilon} \quad(\text { см. обозначения в п. } 3 \S 1), \\
& J_{0}(v)=\frac{1}{2} \int_{\Omega} \bar{\sigma}^{0}(E) E d x-\int_{\Omega} f_{i} v_{i} d x, \quad E=\left\{E_{i j}\right\}, \quad E_{i j}=e_{i j}(v),
\end{aligned}
$$

для $v \in\left(H_{0}^{1}(\Omega)\right)^{3}$, где тензор $\bar{\sigma}^{0}$ определен формулами $(1.17),(1.18)$.

Пусть

$$
\begin{array}{lll}
J_{\varepsilon}=J_{\varepsilon}\left(u^{\varepsilon}\right)=\min J_{\varepsilon}(v) & \text { на множестве } & K_{\varepsilon} \\
J_{0}=J_{0}(u)=\min J_{0}(v) & \text { на множестве }\left(H_{0}^{1}(\Omega)\right)^{3} .
\end{array}
$$


Теорема 3. Энергии $J_{\varepsilon}, J_{0}$ в задачах (2.11), (2.12) связаны мехдуу собой соотношением

$$
\lim _{\varepsilon \rightarrow 0} J_{\varepsilon}=J_{0}
$$

ДоказАТЕЛьство. 1. Докажем, что

$$
\varliminf_{\varepsilon \rightarrow 0} J_{\varepsilon} \geqslant J_{0}
$$

Хорошо известно (см., например, [3; гл. 1]), что минимизанты задач на нахождение минимума $(2.11),(2.12)$ - это функции $u^{\varepsilon}, u$, которые являются решениями вариационного неравенства (1.11) и краевой задачи (1.16) соответственно. Рассмотрим далее тензор $z^{\varepsilon}(x)=\left\{z_{i j}^{\varepsilon}(x)\right\}$, определенный в лемме 5 .

Воспользуемся неравенством Юнга из теории двойственности (см., например, [7; гл. 13]), применяя его к двойственным функционалам

$$
\frac{1}{2} a_{i j k h} e_{i j} e_{k h} \quad \text { и } \frac{1}{2} b_{i j k h} \tau_{i j} \tau_{k h},
$$

определенньм на множестве симметричных тензоров $e=\left\{e_{i j}\right\}$ и $\tau=\left\{\tau_{i j}\right\}$, где тензоры $a=\left\{a_{i j k h}\right\}, b=\left\{b_{i j k h}\right\}$ взаимно обратны и введены в п. $2 \S 1$. Тогда в силу равенств

$$
a e(v) e(v)=a \nabla v \nabla v, \quad z e(v)=z \nabla v
$$

получаем следующую оценку:

$$
\frac{1}{2} a \nabla u^{\varepsilon} \nabla u^{\varepsilon} \geqslant z^{\varepsilon} \nabla u^{\varepsilon}-\frac{1}{2} b z^{\varepsilon} z^{\varepsilon},
$$

где $u^{\varepsilon}$ - минимизант задачи (2.12) и, значит, решение неравенства (1.11). Отсюда для любой подобласти $A \Subset \Omega$ и произвольной функции $\varphi \in C_{0}^{\infty}(A), 0 \leqslant \varphi \leqslant 1$, можем записать

$$
\frac{1}{2} \int_{A} a \nabla u^{\varepsilon} \nabla u^{\varepsilon} d x \geqslant \int_{A} \varphi z^{\varepsilon} \nabla u^{\varepsilon} d x-\frac{1}{2} \int_{A} \varphi b z^{\varepsilon} z^{\varepsilon} d x
$$

слагаемые правой части неравенства (2.14) обозначим через $I_{1, \varepsilon}$ и $I_{2, \varepsilon}$. Тогда в силу леммы 5

$$
\varliminf_{\varepsilon \rightarrow 0} I_{1, \varepsilon} \geqslant \bar{z} \int_{A} \varphi \nabla u^{0} d x \text { при } \varepsilon \rightarrow 0 .
$$

Переходим к пределу при $\varepsilon \rightarrow 0$ в $I_{2, \varepsilon}$, используя свойства среднего значения для периодической функции (см., например, $[7 ;$ гл. $1, \S 1]):$ при $\varepsilon \rightarrow 0$

$$
\begin{aligned}
I_{2, \varepsilon} & \rightarrow-\frac{1}{2} \int_{A} \varphi\left(\int_{Y} b z z d y\right) d x \\
& =-\frac{1}{2} \int_{A} \varphi\left(\int_{Y} a\left(E+e\left(u_{E}\right)\right)\left(E+e\left(u_{E}\right)\right) d y\right) d x=-\frac{1}{2} \int_{A} \varphi \bar{z} E d x,(2.16)
\end{aligned}
$$


где сделаны преобразования в предельном значении на основании свойства (2.3) тензора $z(y)$ из леммы 3 , а также взаимной обратности тензоров $a, b$.

Из (2.14)-(2.16) получаем

$$
\varliminf_{\varepsilon \rightarrow 0} \frac{1}{2} \int_{A} a \nabla u^{\varepsilon} \nabla u^{\varepsilon} d x \geqslant \int_{A} \varphi \bar{z} \nabla u^{0} d x-\frac{1}{2} \int_{A} \varphi \bar{z} E d x
$$

откуда ввиду произвольности функции $\varphi \in C_{0}^{\infty}(A)$ получаем, используя симметричность тензоров $a, e, z$, следующее неравенство:

$$
\varliminf_{\varepsilon \rightarrow 0} \frac{1}{2} \int_{A} a \nabla u^{\varepsilon} \nabla u^{\varepsilon} d x \geqslant \int_{A} \bar{\sigma}^{0}(E) e\left(u^{0}\right) d x-\frac{1}{2} \int_{A} \bar{\sigma}^{0}(E) E d x .
$$

Пусть функция $\omega=\left(\omega_{1}, \omega_{2}, \omega_{3}\right)$ кусочно аффинная, так что $e(\omega)$ - тензор с кусочно постоянными компонентами. Пусть $\left\{A_{j}\right\}$ - подобласти $\Omega$, на которых компоненты $e(\omega)$ постоянны. Для каждой подобласти $A_{j}$ можно получить неравенство типа (2.17). Просуммировав эти неравенства по всем $j$, получаем для произвольной кусочно аффинной функции $\omega$ :

$$
\varliminf_{\varepsilon \rightarrow 0} \frac{1}{2} \int_{\Omega} a \nabla u^{\varepsilon} \nabla u^{\varepsilon} d x \geqslant \int_{\Omega} \bar{\sigma}^{0}(e(\omega)) e\left(u^{0}\right) d x-\frac{1}{2} \int_{A} \bar{\sigma}^{0}(e(\omega)) e(\omega) d x
$$

Поскольку функция $u^{0}$, определенная условием (2.5), оказывается в силу леммы 4 из пространства $\left(H_{0}^{1}(\Omega)\right)^{3}$, то можно найти последовательность кусочно постоянных функций $\left\{\omega^{\delta}\right\}$ такую, что

$$
\int_{\Omega}\left|e\left(\omega^{\delta}-u^{0}\right)\right|^{2} d x \rightarrow 0 \text { при } \delta \rightarrow 0
$$

так как

$$
\|u\|=\left(\int_{\Omega}|e(u)|^{2} d x\right)^{1 / 2}
$$

- одна из возможных норм в пространстве $\left(H_{0}^{1}(\Omega)\right)^{3}$ в силу неравенства Корна.

Заметим, что для выбранной последовательности $\omega^{\delta}$ имеются сходимости: при $\delta \rightarrow 0$

$$
\begin{aligned}
& \int_{\Omega} \bar{\sigma}^{0}\left(e\left(\omega^{\delta}\right)\right) e\left(u^{0}\right) d x \rightarrow \int_{\Omega} \bar{\sigma}^{0}\left(e\left(u^{0}\right)\right) e\left(u^{0}\right) d x \\
& \int_{\Omega} \bar{\sigma}^{0}\left(e\left(\omega^{\delta}\right)\right) e\left(\omega^{\delta}\right) d x \rightarrow \int_{\Omega} \bar{\sigma}^{0}\left(e\left(u^{0}\right)\right) e\left(u^{0}\right) d x
\end{aligned}
$$

Они вытекают из свойства непрерывной зависимости решения вариационного неравенства (1.23) от тензора $E$ в смысле, установленном в лемме 2 , а также из определения тензора $\bar{\sigma}^{0}(E)=\int_{Y} a\left(E+e\left(u_{E}\right)\right) d y$. 
Рассмотрим в (2.18) $\omega=\omega^{\delta}$, перейдем в этом неравенстве к пределу при $\varepsilon \rightarrow 0$ и в результате получим

$$
\begin{aligned}
\frac{\lim _{\varepsilon \rightarrow 0} \frac{1}{2} \int_{\Omega} a \nabla u^{\varepsilon} \nabla u^{\varepsilon} d x}{} & \geqslant \int_{\Omega} \bar{\sigma}^{0}\left(e\left(u^{0}\right)\right) e\left(u^{0}\right) d x-\frac{1}{2} \int_{\Omega} \bar{\sigma}^{0}\left(e\left(u^{0}\right)\right) e\left(u^{0}\right) d x \\
& =\frac{1}{2} \int_{\Omega} \bar{\sigma}^{0}\left(e\left(u^{0}\right)\right) e\left(u^{0}\right) d x .
\end{aligned}
$$

Сходимость

$$
\int_{\Omega} f \cdot u^{\varepsilon} d x \rightarrow \int_{\Omega} f \cdot u^{0} d x \text { при } \varepsilon \rightarrow 0
$$

следует из условия (2.5). Тогда (2.13) вытекает из $(2.20),(2.21)$ и определения функционалов $J_{\varepsilon}\left(u^{\varepsilon}\right), J_{0}\left(u^{0}\right)$, так как

$$
\varliminf_{\varepsilon \rightarrow 0} J_{\varepsilon}=\varliminf_{\varepsilon \rightarrow 0} J_{\varepsilon}\left(u^{\varepsilon}\right) \geqslant J_{0}\left(u^{0}\right) \geqslant \min _{v \in\left(H_{0}^{1}(\Omega)\right)^{3}} J_{0}(v)=J_{0} .
$$

2. Докажем теперь неравенство, противоположное (2.13). Для этого установим следуюший факт: для любой функции $u \in\left(H_{0}^{1}(\Omega)\right)^{3}$ и для любого сколь угодно малого $\delta>0$ сушествует последовательность $\widetilde{u}^{\varepsilon} \in V_{\varepsilon}$ такая, что

$$
\lim _{\varepsilon \rightarrow 0} \int_{\Omega}\left|\tilde{u}^{\varepsilon}-u\right|^{2} d x=0
$$

и

$$
\varlimsup_{\varepsilon \rightarrow 0} \int_{\Omega} a e\left(\widetilde{u}^{\varepsilon}\right) e\left(\widetilde{u}^{\varepsilon}\right) d x \leqslant \int_{\Omega} \bar{\sigma}^{0}(e(u)) e(u) d x+\delta .
$$

В (2.22) можно считать, что $u$-кусочно аффинная функция (к этому приводят те же соображения о непрерывности $\bar{\sigma}^{0}(E)$ по аргументу $E$, которые использовались в п. 1 доказательства теоремы для вывода неравенства (2.20)). Поэтому пусть $\left\{A_{j}\right\}$ - подобласти $\Omega$, на которых компоненты тензора $\left.e(u)\right|_{A_{j}}=E^{(j)}$ постоянны.

Возьмем решения $u^{(j)}$ вариационного неравенства (1.23) с заданным тензором $E=E^{(j)}$. Для него в силу $(2.3)$

$$
\begin{aligned}
\bar{\sigma}^{0}\left(E^{(j)}\right) E^{(j)} & =\int_{Y} a\left(E^{(j)}+e\left(u^{(j)}\right)\right) E^{(j)} d y \\
& =\int_{Y} a\left(E^{(j)}+e\left(u^{j}\right)\right)\left(E^{(j)}+e\left(u^{j}\right)\right) d y .
\end{aligned}
$$

Поэтому положим

$$
\widetilde{u}^{\varepsilon}(x)=u(x)+\left.\varepsilon \Sigma_{j} \varphi_{j}(x) u^{(j)}(y)\right|_{y=x / \varepsilon}, \quad \varphi_{j} \in C_{0}^{\infty}\left(A_{j}\right), \quad 0 \leqslant \varphi_{j} \leqslant 1 ;
$$

более точный выбор $\left\{\varphi_{j}\right\}$ будет указан позже. Тогда

$$
I_{j}^{\varepsilon}=\int_{A_{j}} a \nabla \widetilde{u}^{\varepsilon} \nabla \widetilde{u}^{\varepsilon} d x=\int_{A_{j}} a e\left(\widetilde{u}^{\varepsilon}\right) e\left(\widetilde{u}^{\varepsilon}\right) d x
$$


где

$$
e_{k h}\left(\widetilde{u}^{\varepsilon}\right)=E_{k h}^{(j)}+\varphi_{j} e_{k h, y}\left(u^{(j)}\right)\left(\frac{x}{\varepsilon}\right)+\frac{1}{2} \varepsilon u_{k}^{(j)}\left(\frac{x}{\varepsilon}\right) \frac{\partial \varphi_{j}}{\partial x_{h}}+\frac{1}{2} \varepsilon u_{h}^{(j)}\left(\frac{x}{\varepsilon}\right) \frac{\partial \varphi_{j}}{\partial x_{k}} .
$$

Поэтому, используя вьпуклость подьнтегральной функции в интеграле $I_{j}^{\varepsilon}$, получаем

$$
\begin{aligned}
\varlimsup_{\varepsilon \rightarrow 0} I_{j}^{\varepsilon}= & \left.\varlimsup_{\varepsilon \rightarrow 0} \int_{A_{j}} a\left(E^{(j)}+\varphi_{j} e_{y}\left(u^{(j)}\right)\right)\left(E^{(j)}+\varphi_{j} e_{y}\left(u^{(j)}\right)\right)\right|_{y=x / \varepsilon} d x \\
\leqslant & \varlimsup_{\varepsilon \rightarrow 0}\left[\left.\int_{A_{j}} \varphi_{j} a\left(E^{(j)}+e_{y}\left(u^{(j)}\right)\right)\left(E^{(j)}+e_{y}\left(u^{(j)}\right)\right)\right|_{y=x / \varepsilon} d x\right. \\
& \left.+\int_{A_{j}}\left(1-\varphi_{j}\right) a E^{(j)} E^{(j)} d x\right]
\end{aligned}
$$

где использовано свойство вьпуклой функции: $G(\xi+\varphi \eta) \leqslant \varphi G(\xi+\eta)+(1-\varphi) G(\xi)$, если $G$ - выпуклая функция, $0 \leqslant \varphi \leqslant 1$.

Находим предел при $\varepsilon \rightarrow 0$ от первого слагаемого в квадратной скобке $(2.24)$, используя свойство среднего периодической функции. Тогда из (2.24) получаем

$$
\begin{aligned}
\varlimsup_{\varepsilon \rightarrow 0} I_{j}^{\varepsilon} \leqslant & \int_{Y} a\left(E^{(j)}+e_{y}\left(u^{(j)}\right)\right)\left(E^{(j)}+e_{y}\left(u^{(j)}\right)\right) d y \int_{A_{j}} \varphi_{j} d x \\
& +a E^{(j)} E^{(j)} \int_{A_{j}}\left(1-\varphi_{j}\right) d x=\bar{\sigma}^{0}\left(E^{(j)}\right) E^{(j)} \int_{A_{j}} \varphi_{j} d x \\
& +a E^{(j)} E^{(j)} \int_{A_{j}}\left(1-\varphi_{j}\right) d x \leqslant\left(\bar{\sigma}^{0}\left(E^{(j)}\right) E^{(j)}+\delta_{1}\right)\left|A_{j}\right|,
\end{aligned}
$$

где $\delta_{1}$ - любое наперед заданное малое число, если $\varphi_{j} \in C_{0}^{\infty}\left(A_{j}\right)$ была выбрана соответствуюшим образом.

Суммируя по $j$, получаем из последнего неравенства:

$$
\varlimsup_{\varepsilon \rightarrow 0} \int_{\Omega} a \nabla \widetilde{u}^{\varepsilon} \nabla \widetilde{u}^{\varepsilon} d x \leqslant \Sigma_{j} \varlimsup_{\varepsilon \rightarrow 0} I_{j}^{\varepsilon} \leqslant \int_{\Omega} \bar{\sigma}^{0}(E) E d x+\delta,
$$

где $E=e(u)$, и, таким образом, оценка (2.22) действительно имеет место, если функция $\widetilde{u}^{\varepsilon}$ выбрана по формуле (2.23).

Рассмотрим неравенство (2.22) для функции $u$ - минимизанта задачи (2.12) и любого наперед заданного значения $\delta$. Тогда, имея в виду, что $J_{\varepsilon}=J_{\varepsilon}\left(u^{\varepsilon}\right) \leqslant$ $J_{\varepsilon}\left(\widetilde{u}^{\varepsilon}\right)$, выводим из него, что

$$
\varlimsup_{\varepsilon \rightarrow 0} J_{\varepsilon}=\varlimsup_{\varepsilon \rightarrow 0} J_{\varepsilon}\left(u^{\varepsilon}\right) \leqslant \varlimsup_{\varepsilon \rightarrow 0} J_{\varepsilon}\left(\widetilde{u}^{\varepsilon}\right) \leqslant J_{0}+\delta
$$

где помимо (2.22) использована также очевидная в силу определения (2.23) сходимость

$$
\int_{\Omega} \widetilde{u}^{\varepsilon} \cdot f d x \rightarrow \int_{\Omega} u \cdot f d x \text { при } \varepsilon \rightarrow 0 .
$$

И значит, в силу произвольности $\delta \varlimsup_{\varepsilon \rightarrow 0} J_{\varepsilon} \leqslant J_{0}$, что вкупе с (2.13) доказывает утверждение теоремы 3. 
Теорема 4. Если ${ }^{\varepsilon}, u$ - минимизанты задач (2.11), (2.12) (т.е. решения вариачионного неравенства (1.11) и краевой задачи (1.16) соответственно), mo

$$
\lim _{\varepsilon \rightarrow 0} \int_{\Omega}\left|u^{\varepsilon}-u\right|^{2} d x \rightarrow 0 \quad n p u \quad \varepsilon \rightarrow 0
$$

ДокАЗАТЕЛьСтво. Для минимизанта задачи (2.11) функции $u^{\varepsilon}$ построим приближение $\widetilde{u}^{\varepsilon}$ такое же, как в п. 2 доказательства теоремы 3 , по следующей схеме: сначала для $u$ - минимизанта задачи (2.12) рассмотрим кусочно аффинное приближение в $H^{1}$-метрике $\omega^{\delta}$ и далее для него по формуле $(2.23)$ (где вместо $u$ берем $\omega^{\varepsilon}$ ) сконструируем $\widetilde{u}^{\varepsilon}(\delta)$.

Очевидно равенство для $H^{1}$-нормы рассматриваемых функций:

$$
\left\|\frac{1}{2}\left(u^{\varepsilon}-\widetilde{u}^{\varepsilon}(\delta)\right)\right\|^{2}=\frac{1}{2}\left(\left\|u^{\varepsilon}\right\|^{2}+\left\|\widetilde{u}^{\varepsilon}(\delta)\right\|^{2}\right)-\left\|\frac{1}{2}\left(u^{\varepsilon}+\widetilde{u}^{\varepsilon}(\delta)\right)\right\|^{2},
$$

где $\|w\|=\|w\|_{H^{1}(\Omega)^{3}}$. Заметим, что $\frac{1}{2}\left(u^{\varepsilon}+\widetilde{u}^{\varepsilon}(\delta)\right) \in K_{\varepsilon}$ в силу выбора функций $u^{\varepsilon}, \widetilde{u}^{\varepsilon}(\delta) \in V_{\varepsilon}$ и выпуклости $K_{\varepsilon}$. Поэтому из равномерного неравенства Корна $(1.12)$ для функций из $V_{\varepsilon}$ и ввиду того, что по свойству минимизанта $u^{\varepsilon}$

$$
J_{\varepsilon}=J_{\varepsilon}\left(u^{\varepsilon}\right) \leqslant J_{\varepsilon}\left(\frac{u^{\varepsilon}+\widetilde{u}^{\varepsilon}(\delta)}{2}\right)
$$

получаем

$$
\left\|u^{\varepsilon}-\widetilde{u}^{\varepsilon}(\delta)\right\|^{2} \leqslant C\left(\frac{1}{2} J_{\varepsilon}+\frac{1}{2} J_{\varepsilon}\left(\widetilde{u}^{\varepsilon}(\delta)\right)-J_{\varepsilon}\right) \leqslant C\left(\frac{1}{2} J_{\varepsilon}\left(\widetilde{u}^{\varepsilon}(\delta)\right)-\frac{1}{2} J_{\varepsilon}\right)
$$

откуда следует

$$
\int_{\Omega}\left|u^{\varepsilon}-\widetilde{u}^{\varepsilon}(\delta)\right|^{2} d x \leqslant C_{1}\left(J_{\varepsilon}\left(\widetilde{u}^{\varepsilon}(\delta)\right)-J_{\varepsilon}\right)
$$

где константы $C, C_{1}$ в последних неравенствах не зависят от $\varepsilon, \delta$.

Примем во внимание неравенство

$$
\left\|u^{\varepsilon}-u\right\|_{L^{2}(\Omega)^{3}} \leqslant\left\|u^{\varepsilon}-\widetilde{u}^{\varepsilon}(\delta)\right\|_{L^{2}(\Omega)^{3}}+\left\|\widetilde{u}^{\varepsilon}(\delta)-\omega^{\delta}\right\|_{L^{2}(\Omega)^{3}}+\left\|\omega^{\delta}-u\right\|_{L^{2}(\Omega)^{3}}
$$

Перейдем в нем к пределу сначала при $\delta \rightarrow 0$, потом при $\varepsilon \rightarrow 0$, используя оценку $(2.26)$ и специфику выбора $\omega^{\delta}$ и $\widetilde{u}^{\varepsilon}(\delta)$, а также сходимости: $J_{\varepsilon}\left(\widetilde{u}^{\varepsilon}(\delta)\right) \rightarrow J_{0}$ при $\delta \rightarrow 0, \varepsilon \rightarrow 0$ в силу построения $\widetilde{u}^{\varepsilon}(\delta), J_{\varepsilon} \rightarrow J_{0}$ при $\varepsilon \rightarrow 0$ в силу теоремы 3. В результате заключаем, что справедливо (2.25). Теорема 4 доказана. 


\section{Список литературы}

1. Санчес-Паленсия $E$. Неоднородные среды и теория колебаний. М.: Мир, 1984.

2. Фикера Г. Теоремы сушествования в теории упругости. М.: Мир, 1974.

3. Дюво Г., Лионс Ж.-Л. Неравенства в механике и физике. М.: Наука, 1980.

4. Байокки K., Капело A. Вариационные и квазивариационные неравенства. Приложения к задачам со свободной границей. М.: Наука, 1988.

5. Бахвалов Н. С., Панасенко Г. П. Осреднение процессов в периодических средах. М.: Наука, 1984.

6. Олейник О. А., Иосифьян Г. А., Шамаев А. С. Математические задачи теории сильно неоднородных сред. М.: Изд-во МГУ, 1990.

7. Жиков В.В., Козлов С. М., Олейник О. А. Усреднение дифференциальных операторов. М.: Наука, 1993.

8. Лионс ЖК.-Л. Некоторые методы решения нелинейных краевых задач. М.: Мир, 1972.

9. Жиков В. В. Об усреднении в перфорированных случайных областях общего вида // Матем. заметки. 1993. Т. 53. №1. С. 41-58.

10. Ж Кков В. В. Об усреднении нелинейных вариационных задач в перфорированных областях // Докл. РАН. 1995. Т. 345. № 2. С. 156-160.

11. ЖКиков В. В., Ръчаго М. Е. Усреднение нелинейных эллиптических уравнений второго порядка в перфорированных областях // Изв. РАН. Сер. матем. 1997. Т. 61. №1. C. $69-88$.

12. Zhikov $V$. V. On the homogenization of nonlinear variational problems in perforated domains // Russian J. Math. Phys. 1994. V. 2. №3. P. 393-408.

13. Боговский M. E. Решение первой краевой задачи для уравнения неразрьвности несжимаемой среды // Докл. АН СССР. 1979. Т. 248. № 5. С. 1037-1040.

Московский институт радиотехники, электроники и автоматики (технический университет)
Поступила в редакцию

06.07 .1999 